\title{
Binding Sites of the Viral RNA Element TAR and of TAR Mutants for Various Peptide Ligands, Probed with LILBID: A New Laser Mass Spectrometry
}

\author{
Nina Morgner, ${ }^{a}$ Hans-Dieter Barth, ${ }^{a}$ Bernhard Brutschy, ${ }^{a}$ Ute Scheffer, ${ }^{b}$ \\ Sven Breitung, ${ }^{b}$ and Michael Göbel ${ }^{b}$ \\ a Johann Wolfgang Goethe-Universität Frankfurt Am Main, Institut für Physikalische und Theoretische \\ Chemie, Frankfurt Am Main, Frankfurt am Main, Germany \\ b Johann Wolfgang Goethe-Universität Frankfurt Am Main, Institut für Organische und Chemische Biologie, \\ Frankfurt am Main, Germany
}

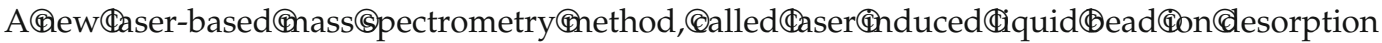
(LILBID),@was@applied@to@investigate@RNA:ligand@interactions.CAs@model@system@the@HIV

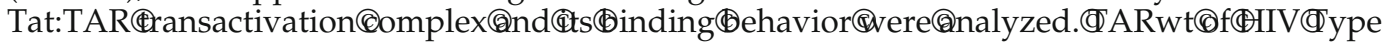

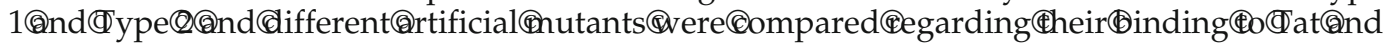
different@eptideđigands.Specific@nd@onspecific@ssociation@o@AR@as@educed,@ith@he bulge@eing@he@vell@known@pecific@inding@ite@f@AR.@n@he@ase@f@riple@rginine@RRR) as@@onspecific@igand, @ultiple@lectrostatic@inding@o@AR@as@ound@t@igher@oncentration@f@RRR.CThis@ontrasted@with@he@ormation@f@nly@ernary@omplexes@n@ompetitive

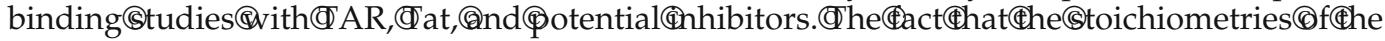
complexes@an@e@etermined@s@@najor@dvantage@f@S@methods@ver@he@videly@pplied fluorimetric@nethods.@@uantitative@valuation@f@he@pectra@y@@umeric@nodel@or@ernary complex@ormation@llows@onclusions@bout@he@ole@nd@trength@f(he@inding@ites@f@he

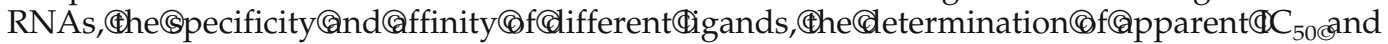

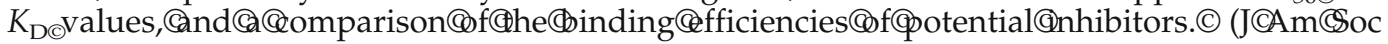

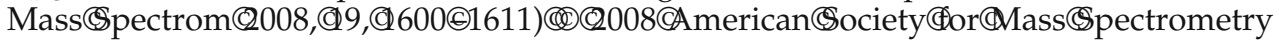

\begin{abstract}
A major@roblem@ssociated@with@inti-HIV@reat-

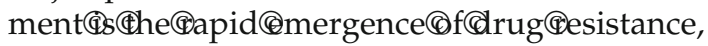
which@s@@erious@mpediment@o@he@ontrol@f HIV@ifections.@Accordingly, @@ompelling@need@s@o discover@nti-HIV@rugs@gainst@alternative@iral@argets.COne@such@target@is@the@HIV@trans-activator@of transcription $₫ T a t) @$ rotein,@which@lays@@eterminant role@n@HIV@eplication@y@epecific@nteraction@with@he trans-activation@esponsive@lement@egion $థ T A R) \Phi 1-5]$. The@atter@s@(s)@ucleotideबRNA@tem-loop@tructure

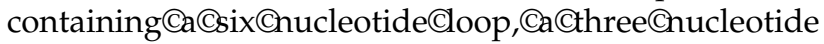
bulge,@nd@wo@ingle-nucleotide@ulges@t $₫$ he(S'@nd@f all@nascently@ranscribed $@ H I V @ m R N A s . C T h e @$ ucleotide@ulge $\left.₫ U_{23} . C_{24}, U_{25}\right) @ f(H I V-1 @ A R @ s @ s s e n t i a l$ for $\odot$ high-affinity $\odot$ binding $(\odot$ to $\odot$ the $\odot$ basic $($ domain

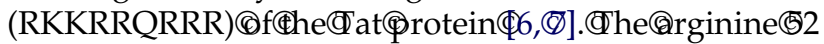

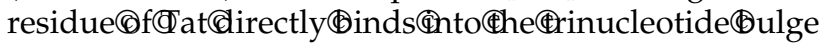
with@ts@uanidinium@roup@eing@argely@esponsible for@heđTat:TAR@interaction@8, ()].đWhile@his@was@1ready®nown@or@ome@ears, $₫$ heđevelopment@fథotent TAR@ligands@is@still@ongoing@and@a@laborious@task
\end{abstract}

Address reprint requests to Professor B. Brutschy, LILBID Mass Spectrometry: Institut für Physikalische und Theoretische Chemie, Johann Wolfgang Goethe-Universität, Max-von-Laue-Strassw 7, 60438 Frankfurt am Main, Germany.®-mail:@rutschy@chemie.uni-frankfurt.de
[10@13].@Suchđigands@would@block@Tat@ransactivation by@nhibition@f@heণTat:TAR@omplex@nd@hus@xert antiviral@ctivity@n@human@ells.

The@rgent@uest@or@uch@inhibitors@s@hallenge@or the@available@screening@methods.@Recent@research@focuses@n@rugs@argeting@he@pecific@inding@f@at@nd TAR@by@such@assays@as@capillary@electrophoresis@or fluorimetric@ssays,@olutionథhase@ootprinting@ia@uorescence@esonance@nergy@ransfer@FRET).@While@ne can@determine $\subseteq K_{D}$ values@with@these $\subseteq$ methods@quite easily, (hey@have@ertain@isadvantages@ince@he@uantitative@valuation@s@ased@n@ssumptions.Che@roblem@s@hat@RET, $₫$ or@xample,@ften@sed@o@etermine binding@onstants,@nly@rovides@he@nformation@f@he labeled@igand@o@bexpelled@ut@f@@pecific@inding pocket(by@n@inhibitor.(However, (4) sites@exist, OtheCformation@ofCspecificCor@nonspecific ternary@omplexes@nay@nduce@imilar@hanges@f@he fluorescence. In this case, the FRET responses give wrong answers and thus the deduced $\mathrm{IC}_{50}$ values are compromised, as is discussed in [14]. Therefore, the use of methods that work without these assumptions and that give direct answers to the question of the stoichiometry of noncovalent complexes might be helpful to understand systems with a more compli- 
cated binding behavior such as the Tat:TAR system. The application of mass spectrometry (MS) to study this specific interaction of Tat with TAR and with potential inhibitors is very desirable due to the inherent advantages of MS such as its versatility, rapidity, and accuracy for mass identification. With mass spectrometry, one can directly determine the drug binding stoichiometry. However, its application to the study of weakly bound noncovalent complexes is still demanding and requires the so-called 3s-properties: softness, sensitivity, and specificity. These requirements are met in differing degrees by existing MS methods introduced in the last decade for bio-MS, such as matrix assisted laser desorption/ionization (MALDI) [15] and electrospray ionization (ESI) [16]. Often, demanding preparation protocols, complex charge state distributions, nonspecific aggregation, ion signal depletion by adduct formation with alkali ions or fragmentation, etc. may complicate the mass spectra [17]. Several studies of the inhibition of Tat:TAR interaction with ESI-MS have been published [18, 19]. Nevertheless, alternative MS techniques may be advantageous in providing new features of information and eventually easier preparation protocols, allowing faster screening as compared to these already well established, and powerful MS methods.

We recently developed a laser desorption mass spectrometry method called laser induced liquid bead ion desorption (LILBID), in which biomolecules are laser desorbed from liquid micro droplets of solution. LILBID [20] proved to be a method of mostly discretionary softness, regarding the sustaining of noncovalent bonds, which allows detection of both specific weakly bound complexes at low laser intensity and at elevated intensity level, and their covalent subunits. For harsh conditions, we use a mildly focused OPO-laser with a pulse energy of 30 $\mathrm{mJ} /$ pulse, and for soft conditions the pulse energy of the laser is reduced to below $2 \mathrm{~mJ} /$ pulse. One of LILBID's positive features is the low analyte consumption of only few microliters of solution at micromolar concentration per analysis, making it an ideal tool for analyzing biomolecules of low availability. This has been demonstrated recently for large membrane molecules [21,22] and for specific complexes of DNA and RNA with ligands [23]. Moreover, the analysis of nucleic acids such as DNA and RNA is possible up to large DNA oligomers such as plasmids [24]. Another important aspect is its tolerance to various buffers in solution, which often play an important role in determining binding selectivity and strength of DNA/RNA-ligand complexes. LILBID in its present form is a development from its earlier version [25-28] using liquid beams, which suffered from a very poor duty cycle.

For this work, all RNA measurements have been performed in the usual LILBID buffer concentrations, which lie in the 5 to $10 \mathrm{mM}$ range, as is usual for ESI measurements as well. First repeated experiments showed that LILBID measurements with $100 \mathrm{mM}$ buffer are more difficult, but possible. The higher ionic strength leads to changes in the binding behavior. Therefore, the following results should be viewed as a proof of principle for the power of the method rather than be interpreted for its biological relevance. Therefore, the apparent $\mathrm{IC}_{50}$ and $K_{\mathrm{D}}$ values are marked by an asterisk in this article.

\section{Experimental}

\section{TAR RNA Mutants}

RNAs were purchased from Biospring (Frankfurt, Germany). HIV-1 TAR RNA 1: 5-GGGCCAGAUCUGAGCCUGGGAGCUCUCUGGCC, TAR bulgeless RNA 2: 5'-GGGCCAGAGAGCCUGGGAGCUCUCUGGCC, HIV-2 TAR RNA 3: 5'- $\mathrm{NH}_{2}-\mathrm{T}_{10}$ GGCCAGAUUGAGCCUGGGAGCUCUCUGGCCT ${ }_{4}$, HIV-1 TAR tetraloop RNA 4: 5'- $\mathrm{NH}_{2}-\mathrm{T}_{10}$ GGCCAGAUCUGAGCUUCGGCUCUCUGGCCT $_{4}$, and TAR bulgeless tetraloop RNA 5: $5^{\prime}-\mathrm{NH}_{2}-\mathrm{T}_{10}$ GGCCAGAGAGCUUCGGCUCUCUGGCC $\mathrm{T}_{4}$. The amino linker and the DNA parts were introduced into RNAs 3-5 to perform experiments not described in this article. All RNAs were purified by HPLC.

\section{Peptides and Peptoids}

Fluorescein-rhodamine-labeled Tat peptide FluoAAARKKRRQRRRAAAC-Rhd 6 was purchased from Thermo Electron Corporation, Ulm, Germany. The tripeptide D-Arg-D-Arg-D-Arg (RRR) 7 was prepared by standard Fmoc solid-phase synthesis and purified by RP-HPLC. The synthesis of peptoids 8 and 9 with the general structure D-Arg-X-D-Arg-CONH${ }_{2}$ will be published elsewhere.

In the subsequent experiments, $K_{\mathrm{D}}{ }^{*}$ and $\mathrm{IC}_{50}{ }^{*}$ values were determined, for which the knowledge of the exact sample concentrations is crucial for getting accurate results. A problem with concentration determination methods is often the missing absolute accuracy, especially to be considered if different methods are used for different classes of molecules. The molar concentrations were always determined with the best method available for a given substance class.

The concentrations of the stock solutions for all RNAs were determined photometrically at $260 \mathrm{~nm}$. With an optical density of $\mathrm{OD}_{260}=1$ corresponding to $40 \mu \mathrm{g} / \mathrm{mL}$, the common conversion factor for RNA was used without consideration of the exact extinction coefficient $\varepsilon$ for every nucleotide. Deviations should be very small and comparable for all RNAs since their sequences are closely related.

Peptides 8 and 9 contain a heterocyclic moiety, which was used for a photometric concentration determination. The extinction coefficient $\varepsilon$ had to be determined first and resulted in the following values: $\varepsilon=37,400$ at $239 \mathrm{~nm}$ for the heterocyclic moiety present in peptide 8 , measured in $50 \mathrm{mM}$ Tris- $\mathrm{HCl}$, $\mathrm{pH} 7.4 ; \varepsilon=29,600$ at $246 \mathrm{~nm}$ for the aminoquinoline 
subunit of peptide 9 , measured in unbuffered aqueous solution.

Concentration determination of Tat peptide 6 was carried out based on the emission of the fluorescein label. After complete digestion of the peptide with proteinase $\mathrm{K}$ (final concentration: $200 \mathrm{ng} / \mu \mathrm{L}$ in $50 \mathrm{mM}$ Tris- $\mathrm{HCl}, 20 \mathrm{mM} \mathrm{KCl}, 0.01 \%$ Triton X-100, pH 7.4), the fluorescence was quantified in a microplate reader (Safire $^{2}$, Tecan 300, Mannedorf, Switzerland) using fluorescein excitation (489 nm) and emission wavelength (525 nm). The result was compared with a calibration curve of fluorescein standards. Because of lacking chromophores in peptide 7 , the concentration was determined gravimetrically. The reliability of the measured value critically depends on the proper drying of the sample.

\section{LILBID}

The detailed experimental setup for LILBID has been published elsewhere [20]. Briefly, microdroplets of solution (diameter $50 \mu \mathrm{m}$ ) are produced on demand at 10 $\mathrm{Hz}$ by a piezo-driven droplet generator (Microdrop, Norderstedt, Germany) and are introduced via a twostage differential pumping from 300 mbar to high vacuum. There the droplets are irradiated one by one by synchronized high-power nanosecond mid-IR laser pulses. A trigger diode is used for the timing of the droplets and the laser pulses. These are generated in a home-built optical parametric oscillator (OPO) using $\mathrm{LiNbO}_{3}$ crystals and a Nd:Yag laser pump. The wavelength of the OPO radiation is tuned to the absorption maximum of water at around $2.94 \mu \mathrm{m}$, corresponding to an excitation of its stretching vibrations. The preformed ions are laser desorbed/ablated from the liquid phase into the gas phase, where ions and counter ions start to recombine due to Coulomb attraction. The "lucky survivors" that escape charge neutralization are mass-analyzed in a reflectron time-of-flight (TOF) mass spectrometer. Both cations and anions may be detected and the charge states of the biomolecules in the mass spectra reflect their charge states in solution, at least in a qualitative way, as discussed in [22]. Thus, ions of nucleic acids appear mostly as anions. The detection limit of LILBID is typically at $\mu$ molar sample concentration. Under this condition, only specific binding effects are to be expected. Therefore, differences in the observed ion distribution should allow conclusions about the character of binding between a RNA and a protein. All presented LILBID measurements were done from typically $5 \mu \mathrm{L}$ of sample solution in a $5 \mathrm{mM}$ ammonium acetate buffer of neutral $\mathrm{pH}$.

\section{FCS}

The FCS measurements are performed as described in [14].

\section{Results and Discussion}

\section{Tat Binding to Different TAR Mutants}

The Tat:TAR complex has been carefully studied by a broad range of methods [6, 7]. To investigate ligand binding by mass spectrometry, it is essential to have a precise knowledge of the transfer function of the mass spectrometric method utilized for detection of noncovalently bonded complexes. Fragmentation and nonspecific complex formation are typical pitfalls in applying MS. It was already shown that at low laser energy LILBID is a very soft method for the detection of noncovalently bound complexes [20]. The RNA:ligand complexes are mainly bound by electrostatic forces, which are further enhanced in the gas phase due to the loss of solvent shielding. Therefore, dissociation of the complexes in the gas phase should be negligible. The desorption process itself is soft enough so that the observed complexes can only be reduced by the use of comparably harsh laser conditions. In the medium or soft desorption range in which the here described experiments were performed, the observed RNA/complexes ratios do not depend on the laser energy. But even when the transfer function is well characterized, distinguishing specific from nonspecific complexation by mass spectrometry alone is a challenge. Usually, it can be done by mutation analysis, i.e., by studying the binding behavior of wild type molecules and some of its mutants and by scrutinizing if the structural changes in the mutants are also reflected in a different binding or dissociation behavior. Therefore, we first examined with LILBID the differences in the binding between a particular Tat peptide $\left(\mathrm{Tat}_{9}, 6\right)$ and the wild type $\mathrm{TAR}_{31}$-RNA fragment and different TAR mutants (Figure 1). These mutants were modified in those regions of the RNA potentially involved in Tat binding, i.e., the bulge, which is a highly specific binding site for Tat, and the loop region, which is assumed to be a possible second binding site. If LILBID is a method suited for the analysis of these complexes, Tat binding should differ for the different mutants, depending on the number of available binding sites.

Lacking the most specific ligand binding region, bulgeless TAR (TARdB) is expected to bind Tat peptide 6 less effectively and less strongly than TAR1wt. Figure $2 b$ and [23] show the LILBID mass spectrum of wild type TAR (TAR1wt) with Tat and, for comparison, Figure $2 \mathrm{a}$ that of TARdB with Tat. Both spectra were recorded at the same molar ratio of 1:1.8 (RNA:Tat). The resulting peak pattern in these two cases is indeed quite different. The experimental ion abundances throughout this article were determined as average integral of each peak area, usually obtained from five to ten spectra per analysis, using the singly charged ion peaks.

In Figure 2a, TARdB and Tat form a 1:1 complex, appearing in the mass spectrum in slightly higher intensity (around 60\%) than the peak of uncomplexed TAR (1:0). Both ions are observed singly and doubly 


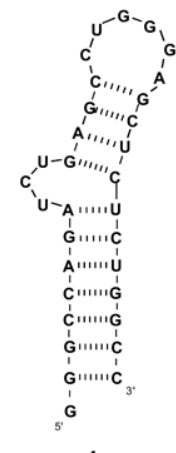

1

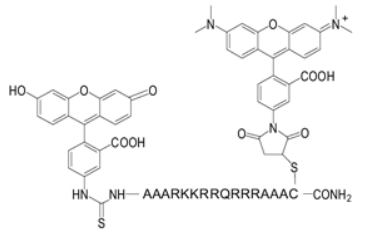

6

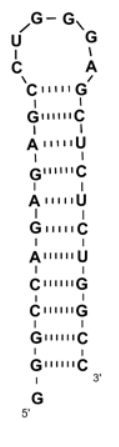

2

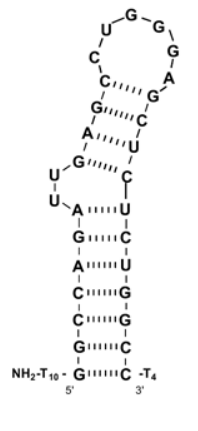

3

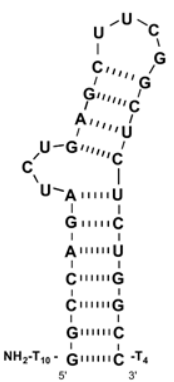

4

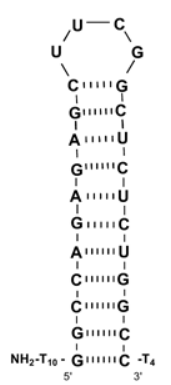

5

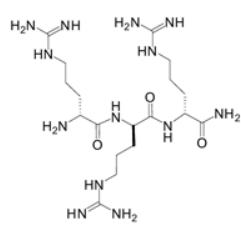

7
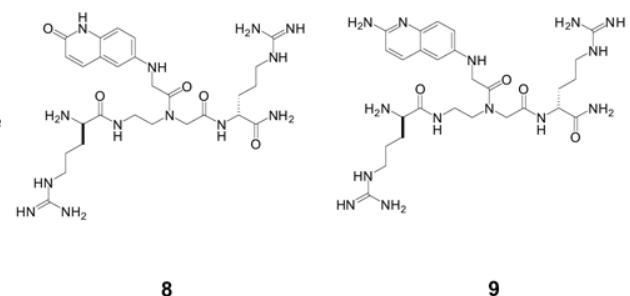

9

Figure 1. Top: Structures of the TAR Models: HIV-1 TAR RNA (TAR1wt) 1, TAR bulgeless RNA (TARdB) 2, HIV-2 TAR RNA (TAR2wt) 3, HIV-1 TAR tetraloop RNA (TAR1dL) 4, and TAR bulgeless tetraloop RNA 5 (TARdBdL). Bottom: Structures of the fluorescein-rhodamine-labeled Tat peptide 6, RFRR 7, and of the synthetic ligands: peptoides R-lactam-R 8 and R-amidine-R 9.

negatively charged. Tat alone is not detected in this anion spectrum, since it is a polycation. With TAR1wt and Tat, on the other hand (Figure 2b), no uncomplexed TAR appears. Compared with bulgeless TAR, the ion distribution for wild type TAR is shifted by the association of one additional Tat peptide, resulting in a distribution of 1:1 and 1:2 complexes (Figure 2a). Thus all TAR1wt bind at least one Tat and about $60 \%$ of them bind a second one. This additional Tat that is bound to $100 \%$ of the TAR1wt must be using the bulge for binding, which is not available in TARbl. At the same time, while the other Tat that binds in almost the same percentage as with TARbl, will use the same binding position as in the bulgeless TAR. This second, probably

(a)

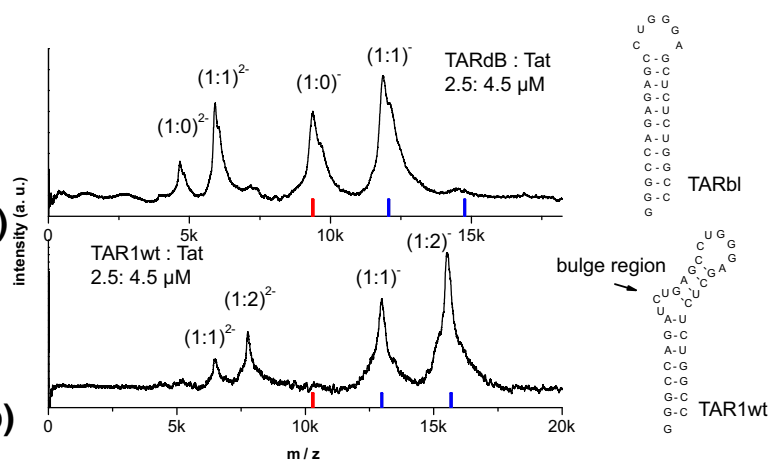

Figure 2. Mass spectra recorded for TARdB (a) and TAR1wt (b) with Tat peptide 6, measured in the anion mode under identical conditions. One additional Tat is bound for the TAR1wt, if compared to the bulgeless version, indicating the specificity of the bulge as binding site. less specific and less strong binding site presumably is localized in the stem or the loop region of the hairpin.

To reinforce these considerations, we want to dwell on the observed charge distributions for a moment. The RNA/ligand systems investigated for this work all show a charge distribution of singly and doubly charged molecules, regardless of the solution net charge varying from 28 to 43 for the different RNAs. This can be explained by the proposed LILBID mechanism, which is understood as a charge neutralization process. High charged molecules are more likely to recombine with counter ions and are therefore more reduced in charge than those ions carrying low charge. So, while the intensity response of LILBID peaks was shown to be linear in a concentration range from $10^{-3}$ to $10^{-7} \mathrm{M}$ for one species [20], the response to solution net charge is more complicated. The spectra merely reflect the solution net charge of the solvated ions, regarding their anionic or cationic character and the order of magnitude of the solution charge state. This is supported by measurements on various types of molecules of different sizes up to a highly charged ribosomal $50 S$ complex with $1.5 \mathrm{MDa}$, which shows a strongly reduced charge distribution of two to twelve [23], even though a more detailed analysis of the response function is yet to be done.

All the RNA/ligand systems investigated here carry charges in the same order of magnitude. The charge differences between the RNA/ligand complexes are therefore not to be expected to lead to nonlinear responses in the ionization process, which would make the comparison and interpretation of the observed ion abundances far more difficult. 
Table 1. Peak areas in \%.

\begin{tabular}{|c|c|c|c|c|c|c|c|c|c|c|c|c|c|c|c|}
\hline \multirow[b]{2}{*}{ Complex mixture } & \multicolumn{3}{|c|}{$\begin{array}{l}\text { TAR1wt } \\
\text { RNA } 1\end{array}$} & \multicolumn{3}{|c|}{$\begin{array}{l}\text { TARdB } \\
\text { RNA } 2\end{array}$} & \multicolumn{3}{|c|}{$\begin{array}{l}\text { TAR2wt } \\
\text { RNA } 3\end{array}$} & \multicolumn{3}{|c|}{$\begin{array}{l}\text { TAR1dL } \\
\text { RNA } 4\end{array}$} & \multicolumn{3}{|c|}{$\begin{array}{l}\text { TARdBdL } \\
\text { RNA } 5\end{array}$} \\
\hline & $1: 0$ & $1: 1$ & $1: 2$ & $1: 0$ & $1: 1$ & $1: 2$ & $1: 0$ & $1: 1$ & $1: 2$ & $1: 0$ & $1: 1$ & $1: 2$ & $1: 0$ & $1: 1$ & $1: 2$ \\
\hline $1: 1.8$ & 0 & 42 & 58 & 29 & $\underline{61}$ & 11 & 30 & $\underline{41}$ & 29 & $\underline{55}$ & 28 & 16 & 28 & $\underline{59}$ & 15 \\
\hline $1: 3.6$ & 0 & 0 & $\overline{100}$ & 19 & $\underline{62}$ & 19 & 18 & $\underline{41}$ & 41 & $\overline{21}$ & $\underline{43}$ & 31 & 13 & $\underline{55}$ & 32 \\
\hline
\end{tabular}

From the obtained results, we conclude that the observed complexation cannot be due to nonspecific electrostatic interactions between the polycation Tat and the polyanionic RNA, since then there should be no differences in the binding behavior between the mutants. Further measurements were recorded with additional TAR mutants at 1:1.8 and 1:3.6 $\mathrm{M}$ ratio of the TAR:Tat binding partners. The relative ion abundances of the mass peaks are listed in Table 1. The TAR variants used (see Figure 1) are the normal HIV-1 wild type TAR (TAR1wt, $\mathbf{1}$ ) as well as HIV-2 TAR (TAR2wt, 3 ). The latter is a wild type TAR sequence from a HIV strain occurring in Africa, differing from TAR1wt by a UU bulge instead of UCU. Additional mutants are with deleted bulge (TARdB, 2), with a stable UUCG-tetraloop (TAR1dL, 4) or with deleted bulge and a tetraloop (TAR$\mathrm{dBdL}, 5)$. The tetraloop variant was used here to prove or rule out the loop as a second binding site for Tat. The loop mutation was chosen in a way to maximally differ from the wt loop while still allowing the formation of a hairpin structure. Tetraloop TAR variants are often preferred for NMR experiments since the modified loop is more stable and conformationally rigid, and the NMR signals therefore more distinct [6].

As summarized in Table 1, the observed binding behavior differs for the different RNAs. In the presence of 3.6 equivalent of Tat peptide 6, TAR1wt is fully converted to the 1:2 complex. Interestingly, no 1:3 or larger stoichiometries were observed. This corroborates the existence of two binding sites for Tat and gives evidence for a high Tat affinity. In contrast, all other mutants show considerably weaker binding of Tat. For each mutant, free RNA is visible in the presence of a 3.6-fold excess of peptide 6. Under such conditions, the $1: 1$ complex is still predominant for TARdB 2. Again, the observed differences between the obtained complex stoichiometries show that we observe liquid phase behavior, since gas-phase attachments would occur independently of the mutations of the RNA.

At this point, it is still an open question whether the specific 1:2 stoichiometry of TAR1wt:Tat is retained with a larger excess of Tat or changes, for example, by nonspecific peptide association. In addition, one has to

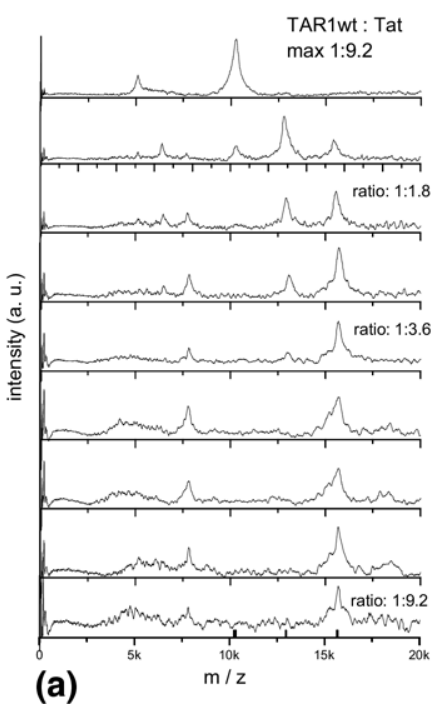

(a)

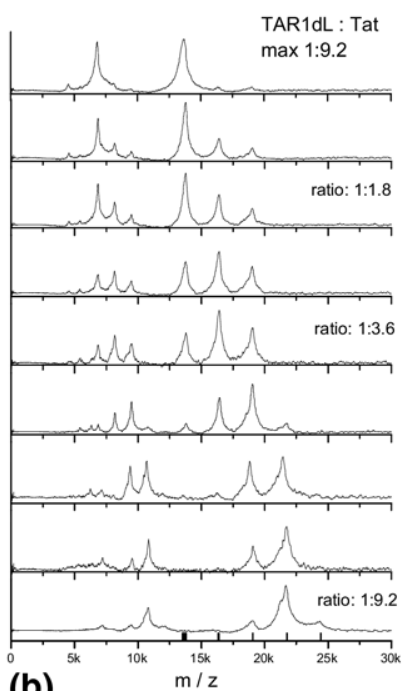

(b)

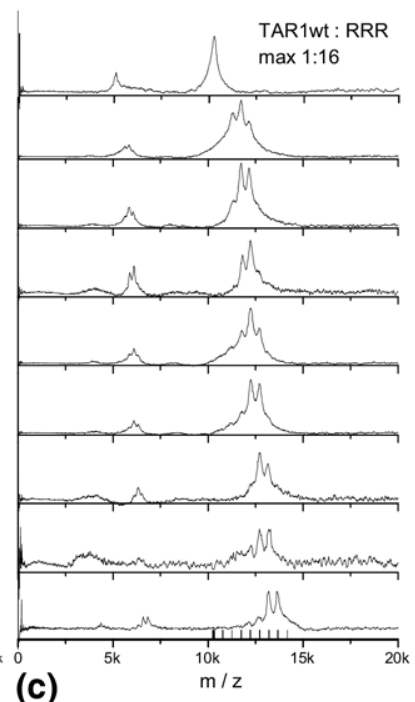

(c)

Figure 3. The specific and nonspecific Tat binding to TAR RNAs is shown in these titration experiments in which the TAR concentration is kept constant at $2.5 \mu \mathrm{M}$ while the Tat concentration is increased to a maximum molar ratio of 1:9.2. The complexes that appear during titration are indicated by lines in every bottom spectrum. The line at the smallest mass represents the uncomplexed RNA. (a) TAR1wt forms specific 1:2 complexes. (b) TAR1dL shows a nonspecific attachment of Tat leading to a statistical distribution of Tat complexes. (c) The same nonspecific binding behavior can be observed for the inhibitor RRR with a TAR:RRR concentration reaching maximally 1:16. The TAR:RRR titrations look the same, independent of the RNA mutant. Shown here is the example of TAR1wt:RRR. The number of attached RRR increases constantly and consistently with the relative RRR concentration. The signal intensity decreases due to neutralization. 
examine if at higher Tat concentration the behavior of the TAR mutants converges to the binding characteristic of TAR1wt. Therefore, we recorded the TAR:Tat complexes by titration with Tat up to a molar ratio of 1:9.2. Figure 3a shows the results for TAR1wt.

The uncomplexed RNA is rapidly displaced by the 1:1 and 1:2 complexes. However, even at large excess of peptide 6, no higher complex stoichiometries are observed. This behavior suggests a sequential association with binding sites of different affinities and excludes further nonspecific attachment of Tat. For TARdB, 9.2 equivalent of Tat still gives $27 \%$ of the $1: 1$ and $73 \%$ of the 1:2 complex. In contrast, TAR2wt exclusively forms 1:2 and 1:3 complexes at elevated Tat concentrations. The highest tendency for 1:3 and even 1:4 stoichiometries was observed with the tetraloop RNAs TAR1dL (data not shown) and TARdBdL (Figure 3b), which show very similar binding behavior under titration of Tat.

It should also be pointed out that at higher Tat concentration, the absolute signal of the cluster ions decreases. This is an effect often observed with LILBID when the attachment of cationic molecules (here: Tat) to anionic molecules (TAR) leads to partial charge compensation in the complexes, which reduces the net charge and hence the escape probability of ions from solution.

From this qualitative comparison it becomes clear that the binding behavior of Tat to TARwt differs noticeably from that of Tat to the mutants. Different relative amounts of Tat are needed to ensure the complexation of RNA. For a quantitative evaluation, we therefore studied titrations of Tat peptide 6 with TAR1wt, TARdB, and TAR1dL in more detail. These experiments were carried out at constant RNA concentrations of $2.5 \mu \mathrm{M}$ with Tat concentrations running from $0.92 \mu \mathrm{M}$ to $23 \mu \mathrm{M}$.

Figure 4 shows the relative intensities of $1: n$ complexes TAR:(Tat) ${ }_{n}(0 \leq n \leq 4)$ depending on the excess of Tat. TAR1wt binds Tat specifically at a high affinity- and then at a low affinity site leading finally to a 1:2 complex (Figure 4a). After the second binding site is saturated, no changes occur upon further addition of Tat. One specific Tat binding site and additional unspecific binding for higher Tat concentrations were observed as well with ESI [29]. TARdB (Figure 4b), on the other hand, shows the same formation of $1: 1$ and 1:2 complexes as TAR1wt but here the curve progressions are flatter and shifted to higher Tat concentrations. The 1:1 complex reaches its maximum at Tat concentrations sufficient to convert TAR1wt completely to the 1:2 complex. No higher complex stoichiometries are observed. We conclude from these differences that there is only one specific and strong binding site in the TAR1wt, namely the bulge, located in the major groove of the duplex. However, a missing bulge leads to some Tat binding as well, though less specific and with lower affinity. Since both RNAs have the loop in common, this region might correspond to the second binding site in
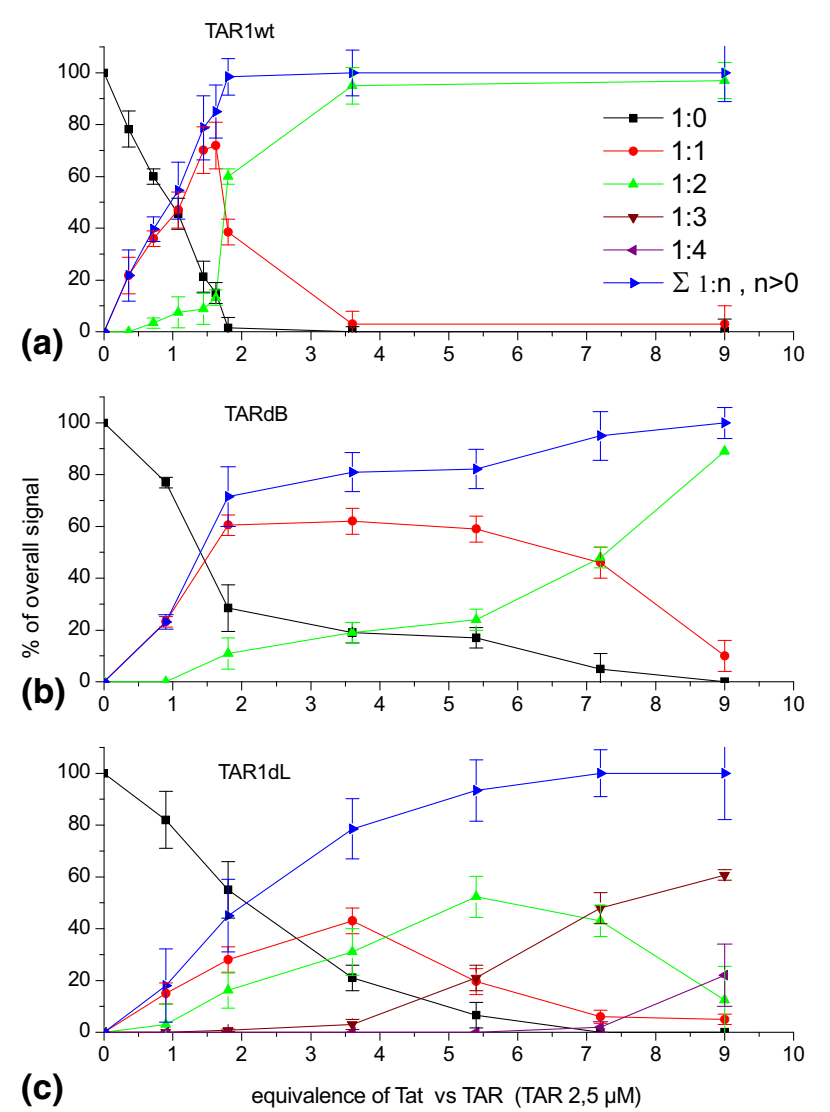

Figure 4. Titration curves of different TAR mutants with Tat. The concentration of the different $1: n$ complexes are shown as a function of the Tat concentration. The TAR concentrations were in each case kept at $2.5 \mu \mathrm{M}$.

TARwt. But we still cannot exclude association in the minor groove, opposite to the bulge. The second copy of Tat, binding TARdB only at higher concentration, might associate in a structurally less defined way, driven mainly by electrostatic interactions. It should be pointed out that both RNAs are not fully neutralized by Tat in the 1:2 complex (maximum charge of Tat peptide 6: +8; maximum charges of TAR1wt and TARdB: -31 and -28 , respectively). The tetraloop RNA TAR1dL, like TARdB, does not show high affinity for Tat peptide 6 (Figure 4c). However, at medium Tat concentrations, the uncomplexed RNA coexists with complexes of the stoichiometries $1: 1,1: 2$, and 1:3. This indicates that the binding in this case must be rather unspecific; otherwise the high affinity of the binding site would lead to an immediate complexation of all RNA before other binding sites are filled. At highest peptide concentrations, $20 \%$ of the RNA forms a 1:4 complex with Tat. TAR1dL bears at maximum 43 negative charges, 28 of them located in the RNA part (Figure 1). In the 1:4 complex, the charge of the RNA part would be overcompensated by Tat. The RNA part clearly cannot harbor four structurally defined Tat binding sites for electrostatic reasons. Thus, 1:4 stoichiometries require binding of the peptide to the single stranded DNA parts of TAR1dL. Alternatively, a complete conversion of its stem-loop 


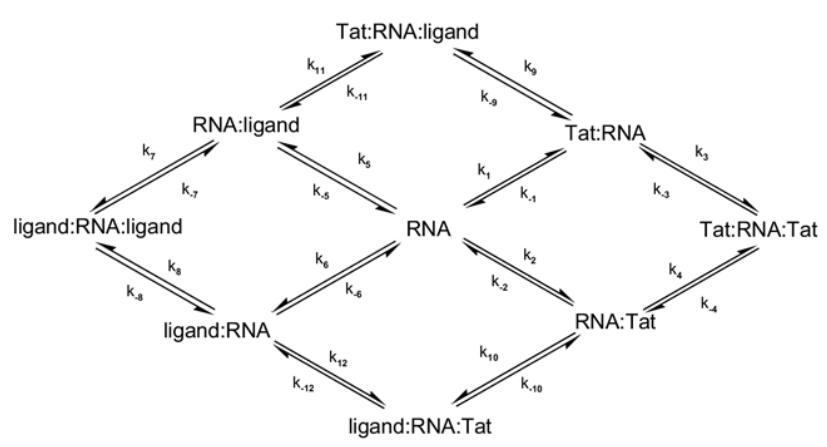

Figure 5. The numeric model used to fit the complex formation is based on second order kinetics (first order for the decay) allowing the depicted reaction pathways.

structure into single strand random coil conformations would allow more copies of Tat to interact with the nucleic acid. It should be kept in mind that even highly stable RNA secondary structures such as TAR may undergo full denaturation when brought in contact with polycations [30].

Tetraloops as present in TAR1dL normally stabilize stem-loop structures in RNA. Why then is denaturation not observed with the hexaloop RNAs TAR1wt and TARdB? The DNA linkers attached to TAR1dL may help to denature the RNA part. A different explanation may be found in subtle stability differences of the TAR-Tat complexes. If Tat interacts with the folded RNA more favorably than with the random coil conformation, thus leading to a stabilized secondary structure, the system will not go beyond 1:2 stoichiometries. The conformational change into a specific structure upon Tat binding has been reported before [6, 31]. In such complexes of TAR1wt, two binding modi might exist [32], where one extended Tat peptide 6 may reside in the major groove (as seen for argininamide [33]) and one in the minor groove (shown for neomycin [34]). If electrostatic interactions with the RNA ligand do not favor the folded conformation, conversion to random coil conformations, and binding of multiple ligands will occur. When TAR1wt is challenged with a large excess of tripeptide 7 (RRR), higher-order complexes close to electrostatic neutrality are found as well (Figure 3c).

For a more detailed analysis, we wanted to fit our data to a quantitative model. The titration experiments shown above allow an easy internal check of relative concentrations of peptide and RNA. In the anion mode, RNA can be observed with or without bound Tat whereas the cationic peptide is not directly visible. Knowing from literature the nanomolar $K_{\mathrm{D}}$ for the Tat:TAR1wt complex [35] and supposing a single binding site, all Tat would be bound to TAR at equimolar conditions. The observed second binding site, on the other hand, requires, at equimolar conditions, that for every 1:2 complex; one copy of TAR has to stay uncomplexed. Higher levels of complexation can only arise from an excess of Tat beyond the 1:1 $\mathrm{M}$ ratio. This consideration allows to calibrate the relative concentra- tions of peptide and RNA, as was done for this study, after the mass spectra of a presumed 1:1 mixture of TAR1wt and Tat revealed the presence of at least 1.8 Tat per RNA. The Tat concentration of the stock solution was scaled accordingly, giving a more reliable relative measure than only the performed concentration determinations with the different described methods could have provided.

Given the two binding sites in TAR1wt and neglecting higher complex stoichiometries, the association of TAR, Tat, and a competitor ligand can be described by a model depicted in Figure 5. To allow a simple numerical treatment of multiple coupled equilibria, each individual $K_{\mathrm{D}}{ }^{*}$ is expressed by a quotient of rate constants $\left(k_{-x} / k_{x}\right)$ describing the forward and backward reaction. From a given start set of concentrations, stepwise calculation of the differential equations is continued till the global equilibrium is reached.

For TAR binding exclusively Tat, only those pathways with rate constants $k_{x}, k_{-x}(1 \leq x \leq 4)$ have to be considered. We were not interested in the rate constants themselves, but in the dissociation constants $K_{\mathrm{D}}{ }^{*}$, given by the ratio $k_{-x} / k_{\mathrm{x}}$. We therefore reduced the number of variables by setting all $k_{-x}$ equal to 1 , a simplification that has no influence on the finally determined $K_{\mathrm{D}}{ }^{*}$ values. Again, the experimental ion abundances were determined as average integral of each peak area. Where higher order complexes occurred, for simplicity their intensities were added on the value for the 1:2 complex. Theoretical complex distributions calculated by the model (Figure 5) were now fitted to the experimental values with rate constants $k_{1}, k_{2}, k_{3}$, and $k_{4}$ used as variables.

This model may be further simplified by assuming the two binding sites to be independent from each other $\left(k_{1}=k_{4}\right.$ and $\left.k_{2}=k_{3}\right)$. Having optimized the two variables in a subsequent second step, all rate constants were allowed to vary independently, starting from the values obtained in the first step to further improve the error of the fit. Hereby the possibility of crosstalk between the first and the second binding site upon the binding of one Tat was included. The resulting numbers in Table 2 shows that the assumption of independent binding sites is not always justified.

In Table 2 , the calculated $K_{\mathrm{D}}^{*}$ values $\left(k_{-\mathrm{x}} / k_{\mathrm{x}}\right)$ are listed. The errors given represent a conservative estimate mainly under consideration of statistical deviations. The $K_{\mathrm{D}}{ }^{*}$ of TAR1wt: Tat is in the low nanomolar

Table 2. Apparent $K_{\mathrm{D}}{ }^{*}$ values $(\mu \mathrm{M} \pm 30 \%)$ as obtained from LILBID measurements for Tat binding to different RNAs under the assumption of the formation of ternary complexes

\begin{tabular}{cccccc}
\hline & $\begin{array}{c}\text { Tat } \\
\text { TAR1wt }\end{array}$ & $\begin{array}{c}\text { Tat } \\
\text { TARdB }\end{array}$ & $\begin{array}{c}\text { Tat } \\
\text { TAR1dL }\end{array}$ & $\begin{array}{c}\text { Tat } \\
\text { TAR2wt }\end{array}$ & $\begin{array}{c}\text { Tat } \\
\text { TARdBdL }\end{array}$ \\
\hline \hline$K_{\mathrm{D}}^{*} 1$ & $<0.01$ & 3.2 & 4 & 3 & 1.5 \\
$K_{\mathrm{D}}{ }^{*} 2$ & 0.1 & 3.2 & 4 & 3 & 9 \\
$K_{\mathrm{D}}{ }^{*} 3$ & 0.15 & 3.2 & 1.5 & 3 & 9 \\
$K_{\mathrm{D}}{ }^{*} 4$ & 0.1 & 3.2 & 1 & 3 & 1.5 \\
\hline
\end{tabular}


range [35], too low to be determined with our experiments running at micromolar concentrations. Thus, although the current data cannot give an accurate $K_{\mathrm{D}}{ }^{*} 1$, we can confirm from our simulations that this value must be in the low $\mathrm{nM}$ range.

With free variation of all four rate constants (without the restrain of keeping $k_{1}=k_{4}$ and $k_{2}=k_{3}$ ) one allows the two binding sites to interact in the model. After the attachment of one multicationic Tat peptide to the polyanionic RNA, the overall charge of the complex will be partly neutralized. Out of electrostatic considerations, one would expect a reduced affinity for the second copy of Tat, the interaction between the two binding Tats being anti-cooperative. Thus, the higher value for $K_{\mathrm{D}}^{*} 4$ than for $K_{\mathrm{D}}^{*} 1$ obtained here appears reasonable, even though the binding mechanism is not fully explained by electrostatics alone. This special binding site with a very low dissociation constant is only observed for TAR1wt.

The $K_{\mathrm{D}}{ }^{*} \mathrm{~s}$ for mutant RNAs are comparable for both simulated binding sites and all lie in the micro molar range. The values for all $\mathrm{dL}$ mutants should not be taken too seriously, since the model ignores the experimentally observed higher-order complexes. Nevertheless $K_{\mathrm{D}}{ }^{*}$ values were estimated for those RNAs based on the obtained mass spectra. Concentrations of peptide 6 that were taken into account were limited to levels not giving 1:3 complexes predominantly. The ion abundances of 1:3 and higher complexes visible in such experiments were added to the 1:2 peaks for the simulation. Therefore, such results can only be taken as a very rough estimate.

\section{Inhibition of Tat Binding}

As noted in the introduction, the inhibition of the Tat:TAR complex could be one way to stop HIV reproduction. It could be suppressed by competitive complex formation with an adequate ligand. To prevent the necessity of high doses, a possible drug should consist of an inhibitor with a dissociation constant $\left(K_{\mathrm{D}}\right)$ preferably lower than that of Tat and, in addition, of high specificity. In this regard, an actual description of the binding competition, which takes place for the Tat:TAR inhibition that utilizes realistic assumptions, can be of great value.

A ligand that is known to bind to RNA is triple arginine (RRR), the minimal variant of the basic binding domain of Tat (RKKRRQRRR) [36]. It interacts with the negatively charged TAR through the positively charged guanidyl residues. From our findings, we can conclude that the binding behavior of RRR is fundamentally different from that of Tat. This can be seen in a titration experiment in which we increased the concentration of RRR continuously up to a molar ratio of 1:16 similar to the titration measurements with TAR and Tat described above. The mass spectra in Figure $3 \mathrm{c}$ show the binding of TAR1wt at increasing excess of RRR. They show a continuous association of RRR to TAR, shifting the peak distribution of complexes to higher masses. As is evident from the (cluster) mass ruler inserted into the mass spectrum at the bottom of Figure $3 c$ representing the theoretical masses of 1:n complexes, the size of the 1:n complexes is shifted continuously from $n=1$ to maximally $n=7$. Interestingly, no broad distribution of those complex sizes is observed, but always a narrow triple of complexes that vary by maximally three RRR. At the same time, the absolute signal intensity decreases, due to the partial neutralization of the complexes similar to the measurement with Tat.

The attachment of up to 7 RRR molecules is only possible by nonspecific electrostatic interaction. Presumably the high RRR concentration leads to a denaturation of the RNA allowing only statistical electrostatic attachment. If a triple arginine, with its three to four positively charged functional groups, binds to a 32 mer TAR fragment having up to 31 negative charges, the association of about 7 to10 RRR molecules should cause charge neutrality if no other ions attach themselves to the RNA. No further increase of $n$ would be expected. Thus, the range of observed cluster sizes supports the assumption of mainly nonspecific electrostatic binding. The appearance of a narrow size distribution is somewhat surprising. It could be rationalized by the assumption that at equilibrium nearly all TAR molecules are more or less equally loaded with RRR. In summary, these data give ample evidence that TAR can be saturated by RRR adsorption.

In contrast to the Tat titrations, the behavior for RRR binding to TAR is independent of a TAR mutation (titration data for other mutants not shown). So in this case, the denaturation cannot be caused by the DNA linkers.

The applicability of a selective inhibitor depends on its displacement efficiency of Tat in the Tat:TAR complex. To test RRR as inhibitor for Tat, a comparative analysis of complex formation was performed. The LILBID mass spectra show complexes of different composition and intensity.

As expected, from the measurements of RRR with TAR alone at low concentration of RRR we now do not observe only species limited in size to ternary complexes, as is typical for specific Tat:TAR binding, but quadruple complexes as well. While additional association of ligand is observed for RRR, which eventually reduces the number of bound Tat in a complex, the maximum number of bound Tat remains two, as expected, from the two specific binding sites of TAR.

A titration experiment was done for which TAR1wt and Tat was kept constant at $2.5 \mu \mathrm{M}$ and the amount of RRR was increased from 0 to $10.5 \mu \mathrm{M}$. To determine an $\mathrm{IC}_{50}{ }^{*}$ value, the observed complex peaks in the mass spectra of the form TAR1wt:Tat ${ }_{n}: \mathrm{RRR}_{\mathrm{m}}$ were grouped into those containing no Tat $(n=0)$, one Tat $(n=1)$, and those with two Tat $(n=2)$. The Tat displacement can therefore be followed as shown in Figure 6b, where the concentration of RRR is plotted against the percentage of the LILBID signal corresponding to complexes con- 


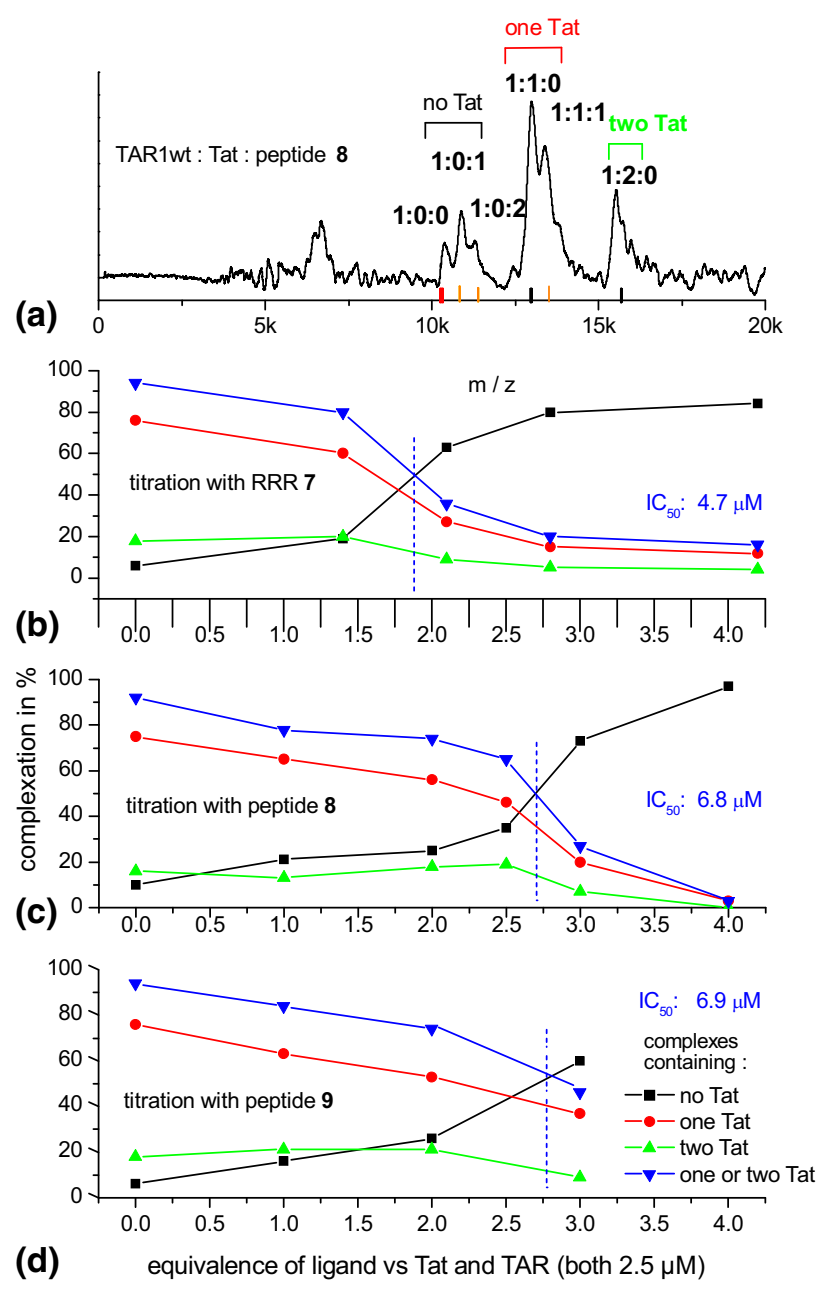

Figure 6. (a) Example mass spectrum for the formation of ternary complexes of the form RNA:(Tat) $n$ :(ligand) $m$. The peaks are grouped for those containing no, one, or two Tat. Titration curves for Tat-TAR inhibitors RRR 7 (b), D-Arg-lactam-D-Arg 8 (c), and D-Arg-amidine-D-Arg 9 (d), could therefore be obtained. The error of the curves is approximated to be $10 \%$.

taining $n=0,1$, or 2 Tat. Additionally, one curve is shown, which represents all RNA that contains Tat (for $n=1$ and $n=2$ ). The amount of bound RRR increases with the RRR concentration. This leads to a decrease in Tat binding, with a maximum of four bound RRR at $10.5 \mu \mathrm{M}$, where only $20 \%$ of the RNA still binds Tat. The RRR concentration, for which the overall complexation of the RNA with Tat drops to $50 \%$ reveals the $\mathrm{IC}_{50}{ }^{*}$ value to be $4.7 \mu \mathrm{M}$.

The inhibition of the TAR1wt Tat complex by RRR was also measured with fluorescence correlation spectroscopy at single molecule level (FCS) by Nandi and coworkers [14]. The $\mathrm{IC}_{50}{ }^{*}$ value determined was $22 \pm 1$ $\mu \mathrm{M}$, while Ludwig et al. determined a value of $4 \mu \mathrm{M}$ with FRET [35]. At high excess concentration of RRR, one must expect nonspecific aggregation, observed as plateau behavior in FCS. Thus, in case of nonspecific aggregation the $\mathrm{IC}_{50}{ }^{*}$ values determined of FCS should be larger than the real one. The FCS and FRET measurements however were performed at higher buffer con- centrations (FCS: $75 \mathrm{mM}$ Tris- $\mathrm{HCl}, 5 \mathrm{mM} \mathrm{MgCl} 2,0.5$ $\mathrm{mg} / \mathrm{mL}$ BSA, pH 7.0, FRET: $50 \mathrm{mM}$ Tris-HCl, $20 \mathrm{mM}$ $\mathrm{KCl}, 0.01 \%$ Triton-X100, $\mathrm{pH} 7.4$ ) so while the results appear in the same order of magnitude, they are not directly comparable. Detailed comparison with LILBID measurements will be done for experiments at higher buffer concentrations.

Another class of ligands using the same binding pockets as Tat might perhaps open the avenue to more efficient inhibiting of Tat binding and thus of HIV replication. For this reason, we tested two small, specially designed ligands (peptides $\mathbf{8}$ and $\mathbf{9}$ ) of comparable size as RRR with a modified binding contact. The structures are shown in Figure 1. Both have a general binding motive of type RXR ( $\mathrm{X}$ being an artificial amino acid) and have been identified as Tat inhibitors in FRET assays. No detailed characterization of the binding stoichiometry has been done so far.

The LILBID mass spectra, which were recorded for TAR1wt and TARdB in equimolar mixture with peptides $\mathbf{8}$ and $\mathbf{9}$, show stoichiometries that are comparable to those measured with Tat. In case of TAR1wt, we see for peptide 8 as well as for peptide 9 the formation of a 1:1 and a 1:2 complex. For TARdB, on the other hand, only the free RNA and 1:1 complex are observed for both ligands. Similar to Tat, the binding behavior obviously depends very much on the existence of the bulge. It indicates that these ligands show similar specificity to TAR as Tat, for the displacement of which they have been designed.

To examine the question if a ligand can compete with Tat for the binding pocket in TAR, as mandatory for an anti HIV drug, a titration experiment was done as for the nonspecific ligand RRR. A typical mass spectrum of a mixture of TAR:Tat:ligand shows complexes of different size and composition (for peptide $\mathbf{8}$ shown in Figure $6 \mathrm{a})$. The $\mathrm{IC}_{50}{ }^{*}$ values determined by titration of two ligands 8 and 9 (see Figure $6 c$ and d) are both almost 7 $\mu \mathrm{M}$ and thus slightly above that of RRR 7 (Figure 6b).

As expected, the two synthetic ligands show a more specific binding behavior as RRR, which attaches in higher number to the RNA. Although the additional attachment of a third ligand is observed in the titration of the ligands at concentrations above $5 \mu \mathrm{M}$, signals corresponding to those complexes are less intense than for RRR and appear only at higher ligand concentration than for RRR. So we can conclude that the ligands have a binding behavior, which resembles that of the specific Tat peptide, though at higher concentration, additional nonspecific association of these small ligands to the RNA occurs as well. As mentioned before, the binding constants of the ligands will depend on experimental conditions such as higher ionic strength or addition of magnesium, which would weaken the complexes. Despite this, we can expect analogous changes for all ligands for which binding is electrostatically dominated. Therefore, the qualitative result from the comparison of $\mathrm{IC}_{50}{ }^{*}$ values for the different ligands will hold as well under more physiological conditions. 
Table 3. Apparent $\mathrm{IC}_{50}$ values (in $\mu \mathrm{M}$ ) obtained by LILBID (errors stemming from standard deviation of the measurements are $\sim 5 \%$ ) and $\mathrm{IC}_{50}$ values (in $\mu \mathrm{M}$ ) from FCS (errors $\sim 5 \%$ )

\begin{tabular}{lccc}
\hline & $\mathrm{IC}_{50}{ }^{*}$ (LILBID) & $\mathrm{IC}_{50}$ (FCS) & Lit. value $^{\mathrm{a}}$ \\
\hline \hline D-Arg-lactam-D-Arg 8 & $6.8 \pm 0.2$ & $5 \pm .25$ & \\
D-Arg-amidine-D-Arg 9 & $6.9 \pm 0.4$ & $3.5 \pm .2$ & \\
D-Arg- D-Arg -D-Arg 7 & $4.7 \pm 0.2$ & $22 \pm 1.1^{\mathrm{b}}$ & 4 \\
\hline
\end{tabular}

${ }^{\text {a } T a k e n ~ f r o m ~[30] . ~}$

${ }^{\mathrm{b}}$ Taken from [28].

A comparison of the obtained $\mathrm{IC}_{50}{ }^{*}$ values of these ligands with RRR (listed in Table 3), determined by FCS, shows that although all values lie in the same concentration range, the agreement of the LILBID data with FCS is better for the more specific synthetic ligands than for the nonspecific RRR, which may be regarded as reasonable.

For the three inhibitors, also $K_{\mathrm{D}}{ }^{*}$ values were determined. As for the more simple interaction of Tat with different TAR mutants the numeric model depicted in Figure 5 was used to calculate equilibrium concentrations of each reaction partner. The calculations were performed in two steps. In a first approximation, all cooperative or anti-cooperative interactions between the two binding sites were neglected. This is done by setting $k_{1}=k_{4}=k_{11}$ for Tat binding to binding site one, $k_{2}=k_{3}=k_{12}$ for Tat binding to binding site two, $k_{6}=k_{7}$ $=k_{10}$ for a ligand binding to binding site one, and $k_{5}=$ $k_{8}=k_{9}$ for a ligand binding to site two. These assumptions were followed as far as possible under the precondition that $k_{1}$ to $k_{4}$ were set to the earlier obtained values from Tat/TAR titration experiments in absence of competitive ligands, as shown in Table 2.

The rate constants were optimized under these assumptions for an error function, calculated from the deviation of the theoretical and the experimental ion signals. Again in a second step, the crosstalk of the binding sites was allowed, by now varying all the $k_{\mathrm{x}}$ values independently, starting from the rate constants obtained before, to further minimize the error of the fit. The resulting $K_{\mathrm{D}}{ }^{*}$ values are summarized in Table 4 .

Interestingly, the $K_{\mathrm{D}}^{*} \mathrm{x}$ values for $\mathrm{x}=1,4,11$ belonging to the strongest Tat binding site are the smallest dissociation constants. The $K_{\mathrm{D}}{ }^{*} 4$ is bigger than $K_{\mathrm{D}}^{*} 1$, which can be rationalized for reasons of electrostatically driven anti-cooperation of the two binding site, as explained in a previous paragraph. $K_{\mathrm{D}}{ }^{*} 11$ describes the binding of a Tat peptide 6 , which binds to a previously formed TAR:ligand complex. The positive charge of the small ligands is noticeably smaller than that of a Tat and therefore can not be expected to have as much of an electrostatic repulsion effect as Tat peptide 6 . Therefore, the anti-cooperative behavior should be less pronounced between Tat and ligand peptides 8 and 9 or between two copies of the tripeptide. This expectation is met by the $K_{\mathrm{D}}{ }^{*}$ values for peptide 8 and $9 . K_{\mathrm{D}}^{*} 9$ and 10 belong to the binding of a small ligand after a Tat binding has already changed the complex into a lower affinity binder. Again, this is an effect that can be observed for peptide 8 and $\mathbf{9}$. If one of these small ligands, which carry fewer charges than a Tat, binds to a RNA, the overall charges are less reduced than by the binding of a Tat. Therefore, the electrostatic situation should not change too much and should be comparable for the binding of a ligand to a RNA or to a RNA:ligand complex. So the $K_{\mathrm{D}}{ }^{*}$ values describing only ligand binding, without an involved Tat, should not differ too much, independent of whether a previous ligand binding might have occurred or not. Therefore, the little variation between $K_{\mathrm{D}}{ }^{*} 5$ to 8 should not be surprising.

$K_{\mathrm{D}}{ }^{*} 12$ is a little lower again, and therefore lies in the same range as those $K_{\mathrm{D}} \mathrm{s}^{*}$ belonging to the binding of the Tat in the less effective binding site; a little higher only by the small effect of an already bound ligand in the other binding site.

One can conclude that the binding of a Tat into a specific binding site also influences the affinity of the other binding site. A conformational change of the RNA upon binding the first Tat might play a role for this as well as electrostatic interactions leading to a less strong second binding site. Therefore, the $K_{\mathrm{D}}{ }^{*}$ values obtained for the ligands peptide 8 and $\mathbf{9}$ seem to be reasonable.

The values for the binding of RRR differ much less from each other, indicating more similar binding sites. The only exceptions are $K_{\mathrm{D}}^{*} 9$ and 10 , which are higher than the other $K_{\mathrm{D}}{ }^{*}$ values for RRR. This is probably due to the same reasons as for the other ligands, though to a noticeably lesser extent. Therefore, the $K_{\mathrm{D}}{ }^{*}$ values found for RRR seem to be less dependent of the binding site, which one might expect from a nonspecific binder. A higher concentration of RRR may lead to a denaturing process of the RNA, which would explain the merely statistical aggregation of RRR in the titration of RRR to any RNA (Figure 5).

The previous considerations show that an interpretation of the obtained data from the mass spectra can go beyond mere qualitative examination. The numeric binding model allowing formation of ternary complexes gave reasonable results, which show that

Table 4. $K_{\mathrm{D}}{ }^{*}$ values (in $\mu \mathrm{M} \pm 30 \%$ ) for the three Tat TAR inhibitors

\begin{tabular}{|c|c|c|c|}
\hline & $\begin{array}{c}\text { D-Arg-lactam- } \\
\text { D-Arg } 8\end{array}$ & $\begin{array}{c}\text { D-Arg-amidine- } \\
\text { D-Arg } 9\end{array}$ & $\begin{array}{c}\text { D-Arg- D-Arg } \\
\text {-D-Arg } 7\end{array}$ \\
\hline$K_{\mathrm{D}}^{*} 1$ & $<0.01$ & $<0.01$ & $<0.01$ \\
\hline$K_{\mathrm{D}}^{*} 2$ & 0.1 & 0.1 & 0.1 \\
\hline$K_{\mathrm{D}}^{*} 3$ & 0.15 & 0.15 & 0.15 \\
\hline$K_{\mathrm{D}} * 4$ & 0.1 & 0.1 & 0.1 \\
\hline$K_{\mathrm{D}}^{*} 5$ & 0.9 & 1 & 0.2 \\
\hline$K_{\mathrm{D}} * 6$ & 1 & 0.6 & 0.2 \\
\hline$K_{\mathrm{D}}^{*} 7$ & 0.5 & 0.5 & 0.1 \\
\hline$K_{\mathrm{D}}^{*} 8$ & 0.9 & 0.9 & 0.2 \\
\hline$K_{\mathrm{D}} * 9$ & 2 & 2 & 0.6 \\
\hline$K_{\mathrm{D}} * 10$ & 2 & 2 & 0.6 \\
\hline$K_{\mathrm{D}} * 11$ & $<0.01$ & $<0.01$ & 0.1 \\
\hline$K_{\mathrm{D}} * 12$ & 0.2 & 0.2 & 0.2 \\
\hline
\end{tabular}


competitive interactions alone are not sufficient to explain the binding situation in a TAR1wt:Tat:ligand system.

For the three inhibitors, the LILBID analysis combined with the numeric model could give a more detailed picture about binding behavior than provided by common fluorimetric methods.

Even though the binding constants of the herescreened ligands have to be improved considerably to inhibit Tat binding quantitatively, evidence is given that they belong to a class of molecules that bind to the same binding sites as Tat. An enhanced binding performance might be achieved for ligands developed from the here investigated small ligands. Such an alternative hybrid ligand would consist of two site-specific ligands interconnected by a spacer of variable length. If each anchors into one specific binding site, the overall $K_{\mathrm{D}}{ }^{*}$ might be considerably lowered and the inhibition of TAR:Tat binding might be more effective.

\section{Conclusions}

Much research has already been invested on compounds binding with high specificity to selected regions of DNA or RNA to accelerate or inhibit reactions. An example is the binding of the Tat:TAR complex, which triggers the translation of HIV genes. Novel inhibitors for this RNA-protein complex might be critical for anti-AIDS strategies. To screen inhibitor candidates quickly and reliably, screening methods are mandatory, which are able to measure either $\mathrm{IC}_{50}{ }^{*}$ values or binding constants and/or to characterize binding specificity, strength, and stoichiometry. Specificity is often studied by mutation analysis of the target and by competitive binding studies, respectively.

LILBID-MS has the potential to become a very useful new MS method for the soft mass analysis of noncovalent complexes containing RNA. Although still of modest mass resolution due to the use of a home-built TOF set-up with no compensation of the initial velocity of the desorbed ions [20], the binding of RNA with ligands can nevertheless be characterized and the binding stoichiometry easily be resolved. The gas-phase ion spectra reveal properties typically observed for the behavior of systems in solution, as is shown here for the example of the well investigated Tat:TAR system with various ligands, for which also FCS studies in solution exist.

The LILBID measurements were all performed in 5 $\mathrm{mM}$ ammonium acetate, which reduces their biological relevance. Experiments at more physiological concentrations of buffer are tolerated by LILBID, as first tests have shown. Though these are more difficult to accomplish, due to instabilities of the droplet generators at higher buffer concentrations, it is a problem that we will overcome by optimized handling procedures. At 100 $\mathrm{mM}$ buffer concentration the observed binding affinities for the second Tat appear reduced. So, while the biological relevant measurements remain a goal for future work, the performed measurements nevertheless show the principle power of the method.

LILBID spectra show differences depending on the TAR mutant, the ligands, and the relative concentrations chosen for the experiments. Important differences in the binding behavior of Tat or the artificial ligands compared with RRR can be ascribed to the different binding character and strength: Specific binding to TAR1wt by Tat or the ligands 8 and $\mathbf{9}$ is deduced from the appearance of complexes binding in only 1:2 stoichiometries, even under excess of the ligand. Existing native binding pockets get filled up, depending on the relative concentration and the $K_{\mathrm{D}}$ of the binding partners. Less specific or even nonspecific binding may also happen and in some cases lead to continuous addition of more and more ligands until finally charge neutrality is reached, as observed for RRR. So, specific and nonspecific binding can be distinguished directly by the comparison of mass spectra taken under varying relative concentrations of the binding partners, which is not easy or even impossible with more standard screening methods such as fluorimetric screening assays, bandshifts, etc., since the formation of ternary or higher complexes can neither be shown nor excluded by these methods.

If different TAR mutants are measured with ligands, mutation-specific binding behavior is only observed in the case of specific ligands. In this case, differences in the spectra can be used to draw conclusions about the targeted binding sites. For unspecific ligands, such as RRR, the results obtained for different mutants are the same.

In titration studies, dissociation constants can be determined, as shown here for the Tat:TAR complex. In case of ternary complex formation "regiospecific" $K_{\mathrm{D}}$ values may be deduced for the various reaction pathways. Quantitative analysis gave evidence of a cross talking of the binding sites often reducing the individual binding constants.

For two ligands, D-Arg-lactam-D-Arg 8 and D-Argamidine-D-Arg 9, it could be shown that they bind specifically to the same binding site of TAR as Tat. Therefore, the design of specific ligands can profit a lot from information that can be obtained by LILBID-MS.

\section{Acknowledgments}

The authors acknowledge support from the Special Research Initiative (SFB 579 RNA Ligand Interaction) of the Deutsche Forschungsgemeinschaft.

\section{References}

1. Péloponèse, J.-M. Jr.; Collette, Y.; Grégoire, C.; Bailly, C.; Campèse, D.; Meurs, E. F.; Olive, D.; Loret, E. P. Full Peptide Synthesis, Purification, and Characterization of Six Tat Variants. J. Biol. Chem. 1999, 27417, 11473-11478

2. Bannwarth, S.; Gatignol, A. HIV-1 TAR RNA: The Target of Molecular Interactions Between the Virus and Its Host. Curr. HIV Res. 2005, 31, 61-71. 
3. Calnan, B.; Tidor, B.; Biancalana, S.; Hudson, D.; Frankel, A. ArginineMediated RNA Recognition: The Arginine Fork. Science 1991, 252(5009), $1167-1171$.

4. Frankel, A. D.; Biancalana, S.; Hudson, D. Proc. Natl. Acad. Sci. U.S.A. 1989, 86, 7397-7401

5. Karn, J. Tackling Tat. J. Mol. Biol. 1999, 293(2), 235-254.

6. Aboul-ela, F.; Karn, J.; Varani, G. The Structure of the Human Immunodeficiency Virus Type-1 TAR RNA Reveals Principles of RNA Recognition by Tat Protein. J. Mol. Biol. 1995, 253(2), 313-332.

7. Weeks, K. M.; Crothers, D. M. RNA Recognition by Tat-Derived Peptides: Interaction in the Major Groove? Cell 1991, 66(3), 577-588.

8. Seewald, M. J.; Metzger, A. U.; Willbold, D.; Rösch, P.; Sticht, H. J. Biomol. Struct. Dyn. 1998, 16, 683-692.

9. Farrow, M. A.; Aboul-ela, F.; Owen, D.; Karpeisky, A.; Beigelman, L.; Gait, M. J. Biochemistry 1998, 37, 3096-3108.

10. Turner, J. J.; Jones, S.; Fabani, M. M.; Ivanova, G.; Arzumanov, A. A.; Gait, M. J. RNA Targeting with Peptide Conjugates of Oligonucleotides, siRNA, and PNA. Blood Cells Mol. Dis. 2007, 38(1), 1-7.

11. Froeyen, M.; Herdewijn, P. RNA as a Target for Drug Design, the Example of Tat-TAR Interaction, Curr. Top. Med. Chem. 2002, 2(10), 1123-1145.

12. Krebs, A.; Ludwig, V.; Boden, O.; Göbel, M. W. Targeting the HIV Trans-Activation Responsive Region-Approaches Towards RNABinding Drugs. Chem. BioChem. 2003, 4(10), 972-978.

13. Richter, S. N.; Palu, G. Inhibitors of HIV-1 Tat-Mediated Transactivation. Curr. Med. Chem. 2006, 13(11), 1305-1315.

14. Nandi, C. K.; Parui, P. P.; Brutschy, B.; Scheffer, U.; Göbel, M. Fluorescence Correlation Spectroscopy at Single Molecule Level on the TatTAR Complex and Its Inhibitors. Biopolymers 2008, 89(1), 17-25.

15. Tanaka, K.; Waki, H.; Ido, Y.; Akita, S.; Yoshida, Y.; Yoshida, T. Protein and Polymer Analyses up to $\mathrm{m} / \mathrm{z} 100,000$ by Laser Ionization Time-ofFlight Mass Spectrometry. Rapid Commun. Mass Spectrom. 1988, 2(8), 151-153.

16. Fenn, J. B.; Mann, M.; Meng, C. K.; Wong, S. F.; Whitehouse, C. M. Electrospray Ionization for Mass Spectrometry of Large Biomolecules. Science 1989, 246, 64-71.

17. Hunger, C. Massenspektrometrische Untersuchungen von DNA, RNA und nichtkovalenten Oligonukleotid-Komplexen. Ph.D. Thesis; Universität Frankfurt am Main, 2005.

18. Mei, H.-Y.; Mack, D. P.; Galan, A. A.; Halim, N. S.; Heldsinger, A.; Loo, J. A.; Moreland, D. W.; Sannes-Lowery, K. A.; Sharmeen, L.; Truong, H. N.; Czarnik, A. W. Bioorg. Med. Chem. 1997, 5, 173-1184.

19. Mei, H.-Y.; Cui, M.; Heldsinger, A.; Lemrow, S. M.; Loo, J. A.; SannesLowery, K. A.; Sharmeen, L.; Czarnik, A. W. Biochemistry 1998, 37, 14204-14212.

20. Morgner, N.; Barth, H.-D.; Brutschy, B. A New Way to Detect Noncovalently Bonded Complexes of Biomolecules from Liquid Micro-Droplets by Laser Mass Spectrometry. Aust. J. Chem. 2006, 59, $109-114$.

21. Meier, T.; Morgner, N.; Matthies, D.; Pogoryelov, D.; Keis, S.; Cook, G. M.; Dimroth, P.; Brutschy, B. A Tridecameric C-Ring of the Adenosine Triphosphate (ATP) Synthase from the Thermoalkaliphilic Bacillus sp. Strain TA2.A1 Facilitates ATP Synthesis at Low Electrochemical Proton Potential. Mol. Microbiol. 2007, 65(5), 1181-1192.

22. Morgner, N.; Kleinschroth, T.; Barth, H.-D.; Ludwig, B.; Brutschy, B. A Novel Approach to Analyze Membrane Proteins by Laser Mass Spectrometry: From Protein Subunits to the Integral Complex. JASMS 2007, $18,1429-1438$.

23. Morgner, N.; Barth, H. D.; Schmidt, T. L.; Heckel, A.; Scheffer, U.; Göbel, M.; Fucini, P.; Brutschy, B. Detecting Specific Ligand Binding to Nucleic Acids: A Test for Ultrasoft Laser Mass Spectrometry. J. Phys. Chem. 2007, 221, 689-704.

24. Hoffmann, J., Morgner, N., Barth, H.-D., Brutschy, B. unpublished results.

25. Sobott, F.; Schunk, S. A.; Schüth, F.; Brutschy, B. Examination of Condensation Products of Group 4 Alkoxides with Laser-Induced Liquid Beam Ionization/Desorption Mass Spectrometry, Chem. Eur. J. 1998, 4, 2353-2359.

26. Kleinekofort, W.; Avdiev, J.; Brutschy, B. A New Method of Laser Desorption Mass Spectrometry for the Study of Biological Macromolecules. Int. J. Mass Spectrom. Ion Processes 1996, 152, 135-142.

27. Kleinekofort, W.; Pfenninger, A.; Plomer, T.; Griesinger, C.; Brutschy, B Observation of Noncovalent Complexes Using Laser-Induced Liquid Beam Ionization/Desorption. Int. J. Mass Spectrom. Ion Processes 1996 156, 195-202.

28. Kleinekofort, W.; Schweitzer, M.; Engels, J.; Brutschy, B. Analysis of Double-Stranded Oligonucleotides by Laser-Induced Liquid Beam Mass Spectrometry. Int. J. Mass Spectrom. Ion Processes 1997, 163, L1-L4.

29. Sannes-Lowery, K. A.; Hu, P . Mack D. P · Mei, H.Y. Loo, J. A. HIV-1 Tat Peptide Binding to TAR RNA by Electrospray Ionization Mass Spectrometry. Anal. Chem. 1997, 69, 5130-5135.

30. Scheffer, U.; Strick, A.; Ludwig, V.; Peter, S.; Kalden, E.; Göbel, M. W Metal-Free Catalysts for the Hydrolysis of RNA Derived from Guanidines, 2-Aminopyridines, and 2-Aminobenzimidazoles. I. Am. Chem. Soc. 2005, 127, 2211-2217.

31. Long, K. S.; Crothers, D. M. Characterization of the Solution Conformations of Unbound and Tat Peptide-Bound Forms of HIV-1 TAR RNA Biochemistry 1999, 38, 10059-10069.

32. Schwiegk, C.; Kalesse, M.; Kirschning, A.; Carlomagno, T. TAR-RNA Recognition by a Novel Cyclic Aminoglycoside Analogue. Nucleic Acids Res. 2006, 34, 3599-3608.

33. Aboul-ela, F.; Karn, J.; Varani, G. The Structure of the Human Immunodeficiency Virus Type-1 TAR RNA Reveals Principles of RNA Recognition by Tat Protein. J. Mol. Biol. 1995, 253, 313-332.

34. Faber, C.; Sticht, H.; Schweimer, K.; Rösch, P. Structural Rearrangements of HIV-1 Tat-Responsive RNA Upon Binding of Neomycin B. J. Biol. Chem. 2000, 275, 20660-20666.

35. Ludwig, V.; Krebs, A.; Stoll, M.; Dietrich, U.; Ferner, J.; Schwalbe, H. Scheffer, U.; Dürner, G.; Göbel, M. W. Tripeptides from Synthetic Amino Acids Block the Tat-TAR Association and Slow Down HIV Spread in Cell Cultures. Chem. Biochem. 2007, 8(15), 1850-1856.

36. Long, K. S.; Crothers, D. M. Interaction of Human Immunodeficiency Virus Type 1 Tat-Derived Peptides with TAR RNA, Biochemistry 1995, $34,8885-8895$. 